\title{
Doses e Horário de Aplicação do Diquat no Controle de Eichhornia crassipes ${ }^{1}$
}

\author{
Effects of Diquat Doses and Spraying Timing on the Control of Eichhornia crassipes
}

\author{
PITELLI, R.A. ${ }^{2}$, BISIGATTO, A.T. ${ }^{3}$, KAWAGUCHI, I. ${ }^{4}$ e PITELLI, R.L.C.M. ${ }^{5}$
}

\begin{abstract}
RESUMO - O aguapé (Eichhornia crassipes) é uma das mais importantes macrófitas aquáticas que colonizam reservatórios e corpos hídricos nas regiões tropicais e subtropicais do mundo, causando uma série de interferências no uso múltiplo da água e do corpo hídrico e no controle de vetores de doenças humanas. Para evitar prejuizos econômicos, sociais, estéticos e ao meio ambiente, essas plantas são controladas por vários métodos, incluindo o controle químico com o uso de herbicidas. Com o objetivo de avaliar a eficácia do herbicida diquat no controle dessa macrófita aquática, foram instalados dois ensaios em casa de vegetação. No primeiro, foram comparadas duas formulações (Reward ${ }^{\circledR}$ e Reglone $($ ) aplicadas em dois horários (diurno e noturno) e em quatro doses $\left(0,1,0,2,0,3\right.$ e 0,4 $\mathrm{kg} \mathrm{ha}^{-1}$ ), num esquema fatorial $2 \times 2 \times 4$, com seis repetições. Os resultados mostraram que a formulação $\operatorname{Reward}{ }^{\mathbb{R}}$ foi mais eficiente no controle dessa macrófita, agindo mais rapidamente, sobretudo em menores doses, e que as aplicações realizadas no período noturno apresentavam melhor desempenho no controle em relação às realizadas no período diurno. Assim, um segundo ensaio foi realizado utilizando apenas a formulação Reward ${ }^{\circledR}$, comparando a aplicação no período noturno e no diurno e as doses de 0,1, 0,2, 0,3 e 0,4 $\mathrm{kg} \mathrm{ha}^{-1}$, obedecendo a um esquema fatorial $2 \times 4$. As plantas de aguapé foram cultivadas em caixas-d'água, e o delineamento experimental adotado foi em blocos ao acaso com quatro repetições, considerando a posição das caixas dentro da casa de vegetação. Os resultados confirmaram que o diquat é eficiente no controle do aguapé, principalmente nas doses de 0,3 e 0,4 $\mathrm{kg} \mathrm{ha}^{-1}$ em aplicações noturnas. Doses baixas e aplicações diurnas não impediram intensas rebrotas das plantas afetadas.
\end{abstract}

Palavras-chave: macrófitas aquáticas, controle químico, aguapé, formulação.

\begin{abstract}
S - Water hyacinth (Eichhornia crassipes) is a major weed in several water bodies in the tropical and sub-tropical regions of the world, causing different types of negative interference in the multiple use of water and water bodies and in the prevention of human diseases. The control of this macrophyte is required under these conditions. Aiming to evaluate the efficacy of the herbicide diquat in the control of water hyacinth, two trials were carried out under greenhouse conditions. In the first assay, a factorial design $2 \times 2 \times 4$ was used comparing two diquat formulations (Reward $\mathbb{R}$ and Reglone ${ }^{\mathbb{R}}$ ), two spraying times (day and night time) and four herbicide doses $(0.1 ; 0.2 ; 0.3$ and $\left.0.4 \mathrm{~kg} \mathrm{ha} \mathrm{h}^{-1}\right)$. The plants were grown in $1 \mathrm{~L}$ pots and the plots were arranged in a completely randomized

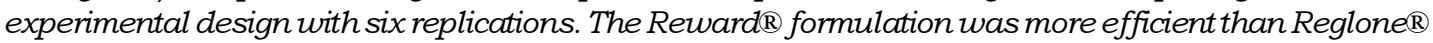
in the control of water hyacinth, acting faster, especially at smaller doses. The night application

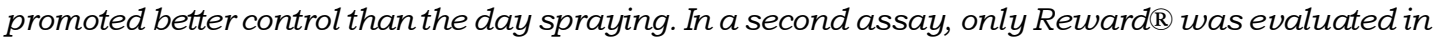
a factorial design experiment comparing two spraying times (day and night time) and four doses $\left(0.1 ; 0.2 ; 0.3\right.$ and $\left.0.4 \mathrm{~kg} \mathrm{ha}^{-1}\right)$. The plants were grown in $40 \mathrm{~L}$ square pots, arranged in a completely randomized block design, with four replications. The results confirmed that night application promoted better control of water hyacinth. The doses of 0.3 and $0.4 \mathrm{~kg} \mathrm{ha-1}$ provided the best control at two months after spraying, preventing intense re-growth of water hyacinth shoots.
\end{abstract}

Keywords: aquatic weeds, chemical control, water hyacinth, formulation.

Recebido para publicação em 5.1.2011 e na forma revisada em 6.5.2011.

2 Professor Titular, Dep. de Fitossanidade, Universidade Estadual Paulista “Júlio de Mesquita Filho” - Unesp, 14884-900 JaboticabalSP. ${ }^{3}$ Engo - -Agr ${ }^{0}$., Unesp Jaboticabal; ${ }^{4}$ Monsanto do Brasil, Tecnologia de Desenvolvimento, São Paulo-SP, ${ }^{5}$ Ecosafe Agricultura e Meio Ambiente Ltda., 14870-410 Jaboticabal-SP. 


\section{INTRODUÇÃO}

As macrófitas aquáticas são muito importantes nas dinâmicas dos diversos tipos de corpos hídricos sempre que ocorrem em tamanhos populacionais compativeis com seus nichos ecológicos. No entanto, em sistemas alterados pelo homem ou quando se trata de plantas exóticas invasoras, desenvolvem grandes populações e podem causar prejuízos ao meio ambiente e aos usos múltiplos da água e do corpo hídrico (Pitelli et al., 2008). Entre as macrófitas aquáticas que causam esses tipos de problema destaca-se o aguapé (Eichhornia crassipes), que é citado em praticamente quase todos os corpos hídricos do Brasil. Embora parte do território brasileiro se insira dentro de sua área de distribuição geográfica, nas regiões leste e sul é considerado uma planta exótica invasora. Na realidade, o aguapé é considerado a mais importante macrófita invasora aquática nas regiões tropical e subtropical do mundo (Holm et al., 1991).

Essa planta é bastante conhecida como importante componente das comunidades de macrófitas aquáticas em reservatórios de hidrelétricas no Brasil, podendo ser citados, como exemplo, os de Bariri (Carvalho et al., 2005; Martins et al., 2008), Barra Bonita (Carvalho et al., 2003; Martins et al., 2008), Porto Primavera (Martins et al., 2009) e Santana (Pitelli et al., 2009). Outros reservatórios citados por Martins et al. (2008) com a presença do aguapé como uma das principais macrófitas aquáticas foram Jaguari, Paraibuna, Chavantes, Jurumirim, Salto Grande, Rosana, Três Irmãos, Mogi-Guaçu, Limoeiro, Água Vermelha, Ibitinga, Promissão e Nova Avanhandava. Em inúmeras situações há necessidade de ações de controle para a redução de suas populações, visando evitar os danos ao meio ambiente e aos usos da água e do reservatório citados em Pitelli et al. (2008).

Entre as opções de manejo, o controle mecânico é o utilizado no Brasil, pois não há estudos relevantes sobre o controle biológico e não há herbicidas registrados, apesar de o controle químico ser adotado em vários países (Pitelli et al., 2008). A ausência de registro no Brasil provavelmente é consequência da existência de poucos trabalhos de pesquisa que evidenciem a eficácia e a segurança ambiental de herbicidas em ambientes aquáticos em condições brasileiras.

No exterior, um dos produtos mais utilizados no controle químico do aguapé é o diquat, um herbicida pertencente ao grupo dos bipiridilos, inibidor do fotossistema I (Rodrigues \& Almeida, 2005), de baixa toxicidade a organismos aquáticos (Henares et al., 2007, 2008a, b,) e sem problemas de biomagnificação (Lavorenti, 1996). Martins \& Pitelli (2005) observaram que a aplicação do diquat para o controle do aguapé ou diretamente sobre a lâmina d'água não promoveu qualquer efeito significativo nas características físicas e químicas da água ao longo do período experimental de 56 dias, quando comparado a tratamentos sem colonização e sem aplicação de diquat. Em outro estudo, Martins (2009) observou que os efeitos da cobertura de $E$. crassipes e da época de avaliação foram os fatores que determinaram as variações nos indices de densidade e de similaridade da comunidade perifitica, sem influência detectável da aplicação do diquat.

No exterior, Jana (1983) observou elevada eficácia do diquat no controle do aguapé. Langeland \& Smith (1993) observaram controle eficiente dessa macrófita com diquat isolado ou combinado com triclopyr. No Brasil, Martins et al. (2002) relataram que o herbicida diquat proporcionou controle de aguapé superior a $90 \%$, onze dias após aplicação de $0,46 \mathrm{~kg} \mathrm{ha}^{-1}$. Neves et al. (2002), em condições de mesocosmos, verificaram que o diquat é eficiente no controle de aguapé a partir da dose de $0,5 \mathrm{~L} \mathrm{ha}^{-1}$, independentemente do horário de aplicação. Em outro ensaio, observaram que aplicações noturnas apenas proporcionaram ação mais rápida do produto, não influenciando o controle final. Comparando aplicações diurnas ou noturnas e em intervalos de 30, 60 e 120 minutos entre a aplicação e a ocorrência de chuva simulada, observaram que no período noturno houve maior importância da extensão do intervalo entre a aplicação do produto e a ocorrência da primeira chuva. Os autores relataram ainda início de rebrotas em alguns tratamentos de controle com o diquat, especialmente em aplicações diurnas. Acessos de aguapé coletados em diferentes locais do Estado de São Paulo apresentaram resposta similar à de doses crescentes de diquat (Cardoso et al., 2003). 
O presente trabalho foi conduzido visando fornecer maiores subsídios para avaliação da ação do diquat no controle de aguapé, adequando doses e horários de aplicação, bem como subsídios para futuras recomendações de controle, após seu registro.

\section{MATERIAL E MÉTODOS}

Dois experimentos foram conduzidos para avaliar o efeito de doses e períodos de aplicação do diquat no controle de Eichhornia crasssipes em casa de vegetação. No primeiro experimento, além das doses e do horário de aplicação, também foram testadas duas formulações do herbicida. Os dois experimentos tiveram alguns procedimentos comuns, que serão relatados a seguir.

As plantas de aguapé foram cultivadas em vasos com sedimento composto por uma mistura do substrato PlantMax ${ }^{\circledR}$ e terra de barranco adubada com 200, 300 e 150 ppm de $\mathrm{N}, \mathrm{P}$ e K, respectivamente (proporção 1:4). A mistura foi complementada com calcário na dosagem de $1,5 \mathrm{~g} \mathrm{~L}^{-1}$. Os vasos foram preenchidos com água até $1 \mathrm{~cm}$ da superficie, deixando uma camada de $10 \mathrm{~cm}$ de água sobre o sedimento. Depois de preparados, os vasos foram incubados por 15 dias antes do plantio do aguapé, para que as reações microaerofilicas ocorressem em nível de sedimento.

No plantio, foram utilizados rebentos de aguapé recém-destacados da planta-mãe, contendo 3-5 folhas, sendo as raízes podadas para estabilização da planta no vaso. O crescimento das plantas de aguapé foi bastante rápido, e a aplicação do herbicida ocorreu aos 35 e 30 dias após o plantio no primeiro e no segundo ensaio, respectivamente.

A aplicação do herbicida foi realizada com um pulverizador costal pressurizado a $\mathrm{CO}_{2}$, munido de barra com quatro bicos XR 11002 trabalhando na pressão de 25 psi, proporcionando um gasto de caldo de $200 \mathrm{~L} \mathrm{ha}^{-1}$.

\section{Primeiro ensaio}

Neste ensaio, os tratamentos foram dispostos em esquema fatorial $2 \times 2 \times 4$, mais duas testemunhas, tendo dois horários de aplicação (diurna e noturna), duas formulações
(Reward ${ }^{\circledR}$ e Reglone ${ }^{\circledR}$ ) e quatro doses de diquat $\left(0,1,0,2,0,3\right.$ e $\left.0,4 \mathrm{~kg} \mathrm{ha}^{-1}\right)$ como as variáveis principais. As duas testemunhas constaram da aplicação de água nos dois horários estabelecidos para a aplicação do herbicida. $\mathrm{O}$ delineamento experimental adotado foi o inteiramente casualizado com seis repetições, devido à possibilidade de movimentação dos vasos dentro da casa de vegetação.

As plantas de aguapé foram cultivadas em caixas plásticas com capacidade de 3,75 litros e dimensões de $15 \times 15 \times 15 \mathrm{~cm}$, sendo plantados dois rebentos por caixa. Por ocasião da aplicação, as plantas e seus rebentos cobriam toda a superfície da caixa.

A aplicação diurna foi realizada entre $10 \mathrm{~h}$ e 10 h25 sob temperatura do ar de $33^{\circ} \mathrm{C}$, temperatura da água de $32{ }^{\circ} \mathrm{C}$, umidade relativa do ar de $44 \%$, cobertura do céu igual a $5 / 8$ e ausência de ventos. A aplicação noturna foi efetuada entre $20 \mathrm{~h} 25$ e $21 \mathrm{~h}$, sob temperatura do ar de $27,5{ }^{\circ} \mathrm{C}$, temperatura da água de $24,5{ }^{\circ} \mathrm{C}$, umidade relativa do ar de $54 \%$, cobertura do céu igual a $3 / 4$ e ventos fracos.

\section{Segundo ensaio}

No segundo ensaio, cinco rebentos de aguapé foram cultivados em caixas de cimento-amianto com dimensões de $0,60 \times 0,60$ x $0,30 \mathrm{~cm}$ de profundidade. Aos 35 dias após o plantio, as plantas e seus novos rebentos cobriam entre 70 e $80 \%$ da superficie das caixas - época em que foram aplicados os tratamentos de controle químico.

Os tratamentos foram dispostos em esquema fatorial $2 \times 4$, mais duas testemunhas, tendo dois horários de aplicação (diurna e noturna) e quatro doses de diquat $(0,1,0,2,0,3$ e $0,4 \mathrm{~kg} \mathrm{ha}^{-1}$ ) como as variáveis principais. As duas testemunhas constaram da aplicação de água nos dois horários estabelecidos para a aplicação do herbicida. O delineamento experimental adotado foi em blocos ao acaso com cinco repetições, sendo os blocos delineados de acordo com a posição relativa da caixa de cimento-amianto na casa de vegetação.

A aplicação diurna foi efetuada entre $13 \mathrm{~h}$ e $13 \mathrm{~h} 45$, sob temperatura de $29,3^{\circ} \mathrm{C}$, temperatura da água de $30,6{ }^{\circ} \mathrm{C}$, umidade relativa do ar de $61 \%$ e ventos fracos $\left(3 \mathrm{~km} \mathrm{~h}^{-1}\right)$. 
A aplicação noturna foi realizada entre $20 \mathrm{~h} 55$ e $21 \mathrm{~h} 13$, sob temperatura do ar de $21^{\circ} \mathrm{C}$, temperatura da água de $22,9{ }^{\circ} \mathrm{C}$, umidade relativa do ar do $90 \%$ e ventos fracos sem rajadas $\left(3 \mathrm{~km} \mathrm{~h}^{-1}\right)$.

\section{Avaliações de controle}

As avaliações foram baseadas na estimativa visual da porcentagem de material vegetal com necrose e morto acima da lâmina de água. Essas estimativas sempre foram realizadas por mais de uma pessoa, sendo utilizada a média dos avaliadores como valor final em cada parcela.

Os dados obtidos em cada avaliação foram submetidos à análise de variância pelo teste $\mathrm{F}$, considerando os diferentes desdobramentos dos graus de liberdade dos tratamentos característicos de cada experimento. Para comparação de média, foi utilizado o teste de Tukey.

\section{RESULTADOS E DISCUSSÃO}

Primeiro ensaio - controle de $E$. crassipes com diquat aplicado em duas formulações, horários e doses

As duas formulações de diquat mostraram eficácias estatisticamente diferentes no controle do aguapé (Tabela 1), sendo o $\operatorname{Reward} \mathbb{R}$ mais eficiente que o Reglone ${ }^{\mathbb{}}$, especialmente por apresentar ação mais rápida (Tabela 2). É possivel observar que as diferenças tiveram alguma expressão nas primeiras datas de avaliação e foram menos expressivas nas avaliações finais. As diferenças estatisticamente significativas nas últimas avaliações podem ser atribuídas aos pequenos coeficientes de variação (Tabela 4).

As diferenças de eficácia de controle para o horário de aplicação como variável principal apenas foram significativas até quatro semanas após aplicação (Tabela 1), sempre com maiores valores de controle para as aplicações noturnas (Tabela 2). A partir da quinta semana, os dois horários de aplicação proporcionaram valores de controle estatisticamente similares.

Em todas as épocas de avaliação houve efeito da dose de diquat no controle de E. crassipes. Os maiores valores foram observados para a dose de $0,4 \mathrm{~kg} \mathrm{ha}^{-1}$, a qual foi estatisticamente superior ao controle proporcionado pela dose de $0,3 \mathrm{~kg} \mathrm{ha}^{-1}$ apenas nas duas primeiras datas de avaliação. Após esse período, o controle proporcionado pelas duas doses foi estatisticamente similar. Em todas as avaliações, as menores duas doses apresentaram valores de controle estatisticamente menores que as doses mais elevadas e também foram diferentes entre si. Aos 28 dias após a aplicação, os valores de controle foram satisfatórios para todas as doses. É interessante observar que, nas menores doses, surgiram pequenos indícios de rebrotas, que poderiam evoluir para a recolonização da planta.

Em nenhuma época de avaliação foi observada interação significativa entre horário e dose de aplicação do diquat (Tabela 1). As interações triplas das variáveis principais

Tabela 1 - Valores de F obtidos na análise de variância de dados de controle do aguapé promovido pelo diquat aplicado em diferentes formulações, doses e horários - primeiro experimento

\begin{tabular}{|c|c|c|c|c|c|c|c|c|c|c|}
\hline \multirow{2}{*}{ Fator de variação } & \multicolumn{10}{|c|}{ Data de avaliação } \\
\hline & 1 & 2 & 3 & 4 & 5 & 6 & 7 & 14 & 21 & 28 \\
\hline $\mathrm{F}$ formulação $(\mathrm{F})$ & $149,45^{* *}$ & $44,65^{* *}$ & $25,29 * *$ & $11,34 * *$ & $8,02 * *$ & $6,61^{*}$ & $9,59 * *$ & $7,82 * *$ & $10,60 * *$ & $7,20 * *$ \\
\hline F horário(H) & $135,65 * *$ & $16,89 * *$ & $8,47 * *$ & $7,80 * *$ & $1,34^{\mathrm{ns}}$ & $0,74^{\mathrm{ns}}$ & $0,28^{\mathrm{ns}}$ & $0,80^{\mathrm{ns}}$ & $0,08^{\mathrm{ns}}$ & $0,70^{\mathrm{ns}}$ \\
\hline $\mathrm{F}$ dose (D) & $142,82 * *$ & $100,21 * *$ & $63,46 * *$ & $51,55 * *$ & $47,19 * *$ & $49,91 * *$ & $79,70 * *$ & $61,63 * *$ & $58,98 * *$ & $33,99 * *$ \\
\hline $\mathrm{F} \mathrm{FxH}$ & $6,06^{*}$ & $2,54^{\mathrm{ns}}$ & $4,81^{*}$ & $5,83 *$ & $5,00 *$ & $3,61^{\mathrm{ns}}$ & $5,21 *$ & $3,41^{\mathrm{ns}}$ & $2,91^{\mathrm{ns}}$ & $1,83^{\mathrm{ns}}$ \\
\hline F F x D & $260,00^{\mathrm{ns}}$ & $6,02 * *$ & $9,52 * *$ & $5,23 * *$ & $4,35^{* *}$ & 2,39 . $^{\mathrm{ns}}$ & $2,05 .^{\mathrm{ns}}$ & $2,80^{\mathrm{ns}}$ & $0,91^{\mathrm{ns}}$ & $0,16^{\mathrm{ns}}$ \\
\hline $\mathrm{F} \mathrm{H} \times \mathrm{D}$ & $0,13^{\mathrm{ns}}$ & $1,96^{\mathrm{ns}}$ & $2,09^{\mathrm{ns}}$ & $1,79^{\mathrm{ns}}$ & $2,14^{\mathrm{ns}}$ & $2,12^{\mathrm{ns}}$ & $2,31^{\mathrm{ns}}$ & $2,30^{\mathrm{ns}}$ & $1,25^{\mathrm{ns}}$ & $0,76^{\mathrm{ns}}$ \\
\hline F F x H x D & $18,60 * *$ & $3,62 *$ & $2,22^{\mathrm{ns}}$ & $3,20 *$ & $3,22 *$ & $3,49 *$ & $6,32 * *$ & $3,57^{*}$ & $1,63^{\mathrm{ns}}$ & $0,81^{\mathrm{ns}}$ \\
\hline $\mathrm{CV}(\%)$ & 5,62 & 5,30 & 5,79 & 5,29 & 5,37 & 5,11 & 3,87 & 4,28 & 4,34 & 5,45 \\
\hline
\end{tabular}

${ }^{*}$ significativo a $5 \%$ de probabilidade; ${ }^{* *}$ significativo a $1 \%$ de probabilidade; ${ }^{\text {ns }}$ não significativo. 
foram significativas nas avaliações realizadas no primeiro, segundo e do quarto ao décimo quarto dias após a aplicação e não serão desdobradas para análise.

A interação entre formulações de diquat e horários de aplicação ocorreu de forma significativa aos 1, 3, 4, 5 e 7 dias após a aplicação (Tabela 1). O desdobramento dos graus de liberdade da interação (Tabela 3) mostrou que na aplicação diurna sempre o Reward $\mathbb{R}$ promoveu controle mais eficaz do aguapé em relação ao Reglone ${ }^{\mathbb{R}}$. Na aplicação noturna essa superioridade do $\operatorname{Reward} \mathbb{R}$ apenas foi observada na primeira avaliação. Considerando apenas os tratamentos que receberam Reglone ${ }^{\circledR}$, a aplicação noturna apenas promoveu controle estatisticamente similar ao da diurna na avaliação realizada aos sete dias após a aplicação. Nas parcelas que receberam a formulação

Tabela 2 - Valores médios de controle de Eichornia crassipes determinados no desdobramento dos graus de liberdade das variáveis principais de formulação, horário de aplicação e dose de diquat - primeiro experimento

\begin{tabular}{|c|c|c|c|c|c|c|c|c|c|c|}
\hline \multirow{2}{*}{ Fator de variação } & \multicolumn{10}{|c|}{ Data de avaliação } \\
\hline & 1 & 2 & 3 & 4 & 5 & 6 & 7 & 14 & 21 & 28 \\
\hline \multicolumn{11}{|c|}{ Efeito da formulação $(\mathrm{F})$} \\
\hline Reglone & $58,00 \mathrm{~b}$ & $70,34 \mathrm{~b}$ & $72,81 \mathrm{~b}$ & $74,75 \mathrm{~b}$ & $75,69 \mathrm{~b}$ & $76,44 \mathrm{~b}$ & $78,06 \mathrm{~b}$ & $85,69 \mathrm{~b}$ & $89,47 \mathrm{~b}$ & $95,06 \mathrm{~b}$ \\
\hline Reward & $73,19 \mathrm{a}$ & $78,50 \mathrm{a}$ & $79,88 \mathrm{a}$ & $79,16 \mathrm{a}$ & $79,41 \mathrm{a}$ & $79,59 \mathrm{a}$ & $81,00 \mathrm{a}$ & $88,19 \mathrm{a}$ & $91,94 \mathrm{a}$ & $96,63 \mathrm{a}$ \\
\hline \multicolumn{11}{|c|}{ Efeito de horário de aplicação $(\mathrm{H})$} \\
\hline Diurna & $58,50 \mathrm{~b}$ & $71,91 \mathrm{~b}$ & $74,41 \mathrm{~b}$ & $75,25 \mathrm{~b}$ & $76,78 \mathrm{a}$ & $77,47 \mathrm{a}$ & $79,25 \mathrm{a}$ & $86,53 \mathrm{a}$ & $90,69 \mathrm{a}$ & $95,63 \mathrm{a}$ \\
\hline Noturna & $72,69 \mathrm{a}$ & $76,94 \mathrm{a}$ & $78,28 \mathrm{a}$ & $78,66 \mathrm{a}$ & $78,31 \mathrm{a}$ & $78,56 \mathrm{a}$ & $79,81 \mathrm{a}$ & $87,34 \mathrm{a}$ & $90,72 \mathrm{a}$ & $96,06 \mathrm{a}$ \\
\hline \multicolumn{11}{|c|}{ Efeito de doses (D) } \\
\hline $0,1 \mathrm{~kg} \mathrm{ha}^{-1}$ & $46,56 \mathrm{~d}$ & $58,13 \mathrm{~d}$ & $62,06 \mathrm{c}$ & $65,31 \mathrm{c}$ & $66,06 \mathrm{c}$ & $66,81 \mathrm{c}$ & $68,88 \mathrm{c}$ & $77,50 \mathrm{c}$ & $81,88 \mathrm{c}$ & $90,06 \mathrm{c}$ \\
\hline $0,2 \mathrm{~kg} \mathrm{ha}^{-1}$ & $62,50 \mathrm{c}$ & $72,75 \mathrm{c}$ & $74,56 \mathrm{~b}$ & $75,19 \mathrm{~b}$ & $76,25 \mathrm{~b}$ & $76,88 \mathrm{~b}$ & $78,38 \mathrm{~b}$ & $86,19 \mathrm{~b}$ & $90,69 \mathrm{~b}$ & $95,94 \mathrm{~b}$ \\
\hline $0,3 \mathrm{~kg} \mathrm{ha}^{-1}$ & $76,13 \mathrm{~b}$ & $80,88 \mathrm{~b}$ & $82,88 \mathrm{a}$ & $82,94 \mathrm{a}$ & $83,00 \mathrm{a}$ & $82,94 \mathrm{a}$ & $84,31 \mathrm{a}$ & $90,63 \mathrm{a}$ & $94,31 \mathrm{a}$ & $98,19 \mathrm{a}$ \\
\hline $0,4 \mathrm{~kg} \mathrm{ha}^{-1}$ & $80,19 \mathrm{a}$ & $85,94 \mathrm{a}$ & $85,88 \mathrm{a}$ & $84,38 \mathrm{a}$ & $84,88 \mathrm{a}$ & $85,44 \mathrm{a}$ & $86,56 \mathrm{a}$ & $93,44 \mathrm{a}$ & $95,94 \mathrm{a}$ & $99,19 \mathrm{a}$ \\
\hline
\end{tabular}

Tabela 3 - Valores médios de controle de Eichhornia crassipes determinados no desdobramento dos graus de liberdade das interações entre as variáveis principais de horário de aplicação e formulação de diquat - primeiro experimento

\begin{tabular}{|l|c|c|c|c|c|c|c|c|c|c|}
\hline \multirow{2}{*}{ Formulação } & \multicolumn{2}{|c|}{ DAA } & \multicolumn{2}{c|}{$3 \mathrm{DAA}$} & \multicolumn{2}{c|}{$4 \mathrm{DAA}$} & \multicolumn{3}{c|}{$5 \mathrm{DAA}$} & \multicolumn{2}{c|}{$7 \mathrm{DAA}$} \\
\cline { 2 - 12 } & Diurna & Noturna & Diurna & Diurna & Diurna & Noturna & Noturna & Noturna & Diurna & Noturna \\
\hline Reglone & $49,38 \mathrm{Bb}$ & $66,63 \mathrm{Ba}$ & $69,44 \mathrm{Bb}$ & $71,63 \mathrm{Bb}$ & $77,88 \mathrm{Aa}$ & $76,19 \mathrm{Aa}$ & $73,63 \mathrm{Bb}$ & $77,75 \mathrm{Aa}$ & $76,88 \mathrm{Ba}$ & $79,25 \mathrm{Aa}$ \\
\hline Reward & $67,63 \mathrm{Ab}$ & $78,75 \mathrm{Aa}$ & $79,38 \mathrm{Aa}$ & $78,88 \mathrm{Aa}$ & $79,44 \mathrm{Aa}$ & $80,38 \mathrm{Aa}$ & $79,94 \mathrm{Aa}$ & $78,88 \mathrm{Aa}$ & $81,63 \mathrm{Aa}$ & $80,38 \mathrm{Aa}$ \\
\hline
\end{tabular}

Letras maiúsculas comparam médias para formulação dentro dos horários de aplicação; letras minúsculas comparam médias para horários de aplicação dentro de formulações de diquat. Médias acompanhadas de mesma letra não diferem estatisticamente entre si.

Tabela 4 - Valores médios de controle de Eichhornia crassipes determinados no desdobramento dos graus de liberdade das interações entre as variáveis principais de doses e formulação de diquat - primeiro experimento

\begin{tabular}{|c|c|c|c|c|c|c|c|c|}
\hline \multirow{2}{*}{ Dose de diquat } & \multicolumn{2}{|c|}{$2 \mathrm{DAA}$} & \multicolumn{2}{|c|}{$3 \mathrm{DAA}$} & \multicolumn{2}{|c|}{$4 \mathrm{DAA}$} & \multicolumn{2}{|c|}{$5 \mathrm{DAA}$} \\
\hline & Reglone $\AA$ & Reward $囚$ & Reglone $\AA$ & Reward® & Reglone $\AA$ & Reward $囚$ & Reglone ${ }^{\circledR}$ & Reward $\AA$ \\
\hline $0,1 \mathrm{~kg} \mathrm{ha}^{-1}$ & $52,96 \mathrm{Db}$ & $63,31 \mathrm{Ca}$ & $58,82 \mathrm{Db}$ & $65,31 \mathrm{Ca}$ & $59,32 \mathrm{Ca}$ & $71,31 \mathrm{Bb}$ & $60,8 \mathrm{~B}$ & $71,32 \mathrm{~B}$ \\
\hline $0,2 \mathrm{~kg} \mathrm{ha}^{-1}$ & $69,7 \mathrm{Cb}$ & $75,82 \mathrm{Ba}$ & $68,92 \mathrm{Cb}$ & $80,23 \mathrm{Ba}$ & $77,25 \mathrm{Ba}$ & $73,12 \mathrm{Bb}$ & $75,38 \mathrm{AB}$ & $77,12 \mathrm{~B}$ \\
\hline $0,3 \mathrm{~kg} \mathrm{ha}^{-1}$ & $77,06 \mathrm{Bb}$ & $84,72 \mathrm{Aba}$ & $78,96 \mathrm{Bb}$ & $86,86 \mathrm{Aa}$ & $81,12 \mathrm{Aa}$ & $84,71 \mathrm{Aa}$ & $82,68 \mathrm{~A}$ & $83,32 \mathrm{~A}$ \\
\hline $0,4 \mathrm{~kg} \mathrm{ha}^{-1}$ & $81,68 \mathrm{Aa}$ & $90,21 \mathrm{Aa}$ & $84,66 \mathrm{Aa}$ & $87,15 \mathrm{Aa}$ & $81,11 \mathrm{Aa}$ & $87,64 \mathrm{Aa}$ & $83,73 \mathrm{~A}$ & $86,03 \mathrm{~A}$ \\
\hline
\end{tabular}

Letras maiúsculas comparam médias para formulação dentro dos horários de aplicação; letras minúsculas comparam médias para horários de aplicação dentro de formulações de diquat. Médias acompanhadas de mesma letra não diferem estatisticamente entre si. 
Reward ${ }^{\circledR}$ praticamente não foram verificados efeitos de horários de aplicação, ocorrendo diferença estatística apenas na avaliação realizada no primeiro dia após a aplicação.

A interação entre as formulações e doses de diquat foi significativa para as avaliações realizadas aos 2, 3, 4 e 5 dias após a aplicação (Tabela 1). Os desdobramentos das interações mostraram que o Reward $\mathbb{R}$ foi mais eficaz no controle do aguapé que o Reglone ${ }^{\circledR}$ nas três doses mais baixas, nas avaliações realizadas aos dois e três dias após a aplicação. No quarto dia, a superioridade estatística do Reward $\mathbb{R}$ apenas foi observada nas duas doses mais baixas e, no quinto dia de avaliação, apenas na dose de $0,1 \mathrm{~kg} \mathrm{ha}^{-1}$.

Os resultados desse primeiro ensaio mostraram que a formulação Reward $\mathbb{R}$ foi mais adequada para o controle do aguapé, tendo ação mais rápida e agindo com eficácia em doses menores, e que aplicações noturnas proporcionam melhores valores de controle que as realizadas durante o dia. Também ficou evidente que o período de observações foi pequeno, pois houve apenas evidências de rebrota da macrófita. Desse modo, no segundo ensaio foi

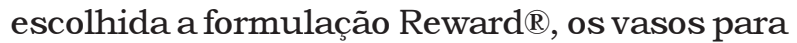
o crescimento do aguapé foram maiores e o período de avaliações foi mais extenso.

\section{Segundo ensaio - controle de $E$. crassipes com diquat aplicado em diferentes horários e doses}

A análise de variância dos dados de controle do aguapé (Tabela 5) mostrou que os blocos não diferiram significativamente entre si, indicando que a posição dos vasos na casa de vegetação não influenciou a ação de controle promovida pelo diquat nessa macrófita. Os efeitos de doses foram significativos para todas as épocas de avaliação, e os efeitos do horário de aplicação apenas foram significativos a partir de avaliação realizada aos 19 dias após a aplicação. Os efeitos interativos entre o horário da aplicação e a dose do diquat no controle do aguapé apenas foram significativos a partir da avaliação realizada aos 74 dias após a aplicação.

O teste de comparação das médias das variáveis principais (Tabela 6) mostrou que em todas as épocas de avaliação o controle do aguapé proporcionado pelo diquat foi superior quando a aplicação foi noturna. Aos 6 e 47 dias após a aplicação, o controle proporcionado pela aplicação noturna foi 9-19\% superior ao da aplicação diurna. Nas duas últimas épocas de avaliação essa diferença cresceu para 33 e $43 \%$, respectivamente. Esse resultado é decorrente das reduções dos valores de controle observados para a aplicação diurna, devido ao aparecimento e crescimento de rebrotas nas plantas. Esse comportamento foi muito mais evidente nos tratamentos com aplicação diurna, cujo valor máximo de controle foi de $91 \%$ aos 40 dias após a aplicação, o qual foi reduzido para $64 \%$ aos 61 dias. No grupo de tratamentos que receberam aplicação noturna, o máximo valor médio de controle também foi observado aos 40 dias após a aplicação: 99\%. Neste grupo a rebrota foi menos intensa, e aos 61 dias o valor médio de controle foi de $94 \%$.

$\mathrm{O}$ diquat apresentou elevada porcentagem de controle do aguapé, a qual foi relacionada à dose do produto. Sempre as melhores avaliações de controle foram observadas na dose de $0,4 \mathrm{~kg} \mathrm{ha}^{-1} \mathrm{e}, \operatorname{dos} 12$ aos 54 dias após a aplicação, os seus valores foram estatisticamente similares aos observados para as doses de 0,2 e $0,3 \mathrm{~kg} \mathrm{ha}^{-1}$, mas sempre superiores aos observados na menor dose $\left(0,1 \mathrm{~kg} \mathrm{ha}^{-1}\right)$. As reduções dos valores de controle do aguapé pela rebrota das plantas ocorreram em todas as doses testadas. Nas doses de 0,1, 0,2 e $0,4 \mathrm{~kg} \mathrm{ha}^{-1}$ as melhores avaliações de controle foram observadas aos 40 dias após a aplicação, com valores de 87, 97 e $87 \%$, respectivamente. Aos 61 dias, os valores de controle foram de 54,78 e $97 \%$, respectivamente. Na dose de $0,3 \mathrm{~kg} \mathrm{ha}^{-1}$ o maior valor de controle, $97 \%$, foi observado aos 47 dias após a aplicação e foi reduzido para $88 \%$ aos 61 dias. Os resultados mostram que o controle máximo médio obtido foi similar para as três maiores doses, porém a intensidade de rebrota foi reduzida à medida que a taxa de aplicação cresceu, ou seja, há maior rebrota do aguapé em menores doses do diquat.

Na Tabela 7 estão apresentados os valores médios de controle de Eichhornia crassipes obtidos no desdobramento dos graus de liberdade da interação entre doses de diquat e horário de aplicação para as avaliações realizadas 
Tabela 5 - Valores de F obtidos na análise de variância de dados de controle do aguapé promovido pelo diquat aplicado em diferentes doses e horários - segundo experimento

\begin{tabular}{|c|c|c|c|c|c|c|c|c|c|}
\hline \multirow{2}{*}{ Variáveis } & \multicolumn{9}{|c|}{ Época de avaliação } \\
\hline & 6 DAA & $12 \mathrm{DAA}$ & $19 \mathrm{DAA}$ & $26 \mathrm{DAA}$ & 33 DAA & 40 DAA & $47 \mathrm{DAA}$ & $54 \mathrm{DAA}$ & $61 \mathrm{DAA}$ \\
\hline F blocos & $0,52^{\mathrm{ns}}$ & $0,25^{\mathrm{ns}}$ & $0,22^{\mathrm{ns}}$ & $0,23^{\mathrm{ns}}$ & $0,36^{\mathrm{ns}}$ & $0,10^{\mathrm{ns}}$ & $0,23^{\mathrm{ns}}$ & $0,30^{\mathrm{ns}}$ & $0,48^{\mathrm{ns}}$ \\
\hline F doses & $19,08^{* *}$ & $6,23 * *$ & $12,83 * *$ & $10,94 * *$ & $10,50 * *$ & $7,73 * *$ & $12,33 * *$ & $18,95 * *$ & $15,94 * *$ \\
\hline F horário & $4,14^{\mathrm{ns}}$ & $1,90^{\mathrm{ns}}$ & $14,64 * *$ & $11,67 * *$ & $23,96 * *$ & $15,95 * *$ & $25,04 * *$ & $38,43 * *$ & $34,10 * *$ \\
\hline $\mathrm{F} \mathrm{H} \times \mathrm{D}$ & $0,51^{\mathrm{ns}}$ & $0,23^{\mathrm{ns}}$ & $1,77^{\mathrm{ns}}$ & $1,35^{\mathrm{ns}}$ & $2,02^{\mathrm{ns}}$ & $1,57^{\mathrm{ns}}$ & $3,37^{*}$ & $6,05 * *$ & $7,00 * *$ \\
\hline F H d. $0,1 \mathrm{~kg} \mathrm{ha}^{-1}$ & - & & - & - & - & - & $24,45 * *$ & $41,13 * *$ & $34,85 * *$ \\
\hline F H d. $0,2 \mathrm{~kg} \mathrm{ha}^{-1}$ & - & - & - & - & - & - & $7,90^{*}$ & $11,80 * *$ & $17,45^{* *}$ \\
\hline F H d. $0,3 \mathrm{~kg} \mathrm{ha}^{-1}$ & - & - & - & - & - & - & $2,23^{\mathrm{ns}}$ & $3,00^{\mathrm{ns}}$ & $2,76^{\mathrm{ns}}$ \\
\hline F H d. $0,4 \mathrm{~kg} \mathrm{ha}^{-1}$ & - & - & - & - & - & - & $0,58^{\mathrm{ns}}$ & $0,67^{\mathrm{ns}}$ & $0,00^{\mathrm{ns}}$ \\
\hline F D d. Diurna & - & - & - & - & - & - & $14,23 * *$ & $22,93 * *$ & $21,64 * *$ \\
\hline F D d. Noturna & - & - & - & - & - & - & $1,47^{\mathrm{ns}}$ & $2,07^{\mathrm{ns}}$ & $1,29^{\mathrm{ns}}$ \\
\hline $\mathrm{CV}(\%)$ & 8,14 & 12,77 & 9,86 & 9,82 & 7,89 & 7,58 & 7,83 & 9,52 & 13,20 \\
\hline
\end{tabular}

* significativo a $5 \%$ de probabilidade; $* *$ significativo a $1 \%$ de probabilidade; ns não significativo.

Tabela 6 - Valores médios de controle de Eichhornia crassipes determinados no desdobramento dos graus de liberdade das variáveis principais de horário de aplicação e dose de diquat - segundo experimento

\begin{tabular}{|c|c|c|c|c|c|c|c|c|c|}
\hline \multirow{2}{*}{ Fator de variação } & \multicolumn{9}{|c|}{ Dias após a aplicação } \\
\hline & 6 DAA & 12 DAA & 19 DAA & $26 \mathrm{DAA}$ & 33 DAA & 40 DAA & $47 \mathrm{DAA}$ & 54 DAA & $61 \mathrm{DAA}$ \\
\hline \multicolumn{10}{|c|}{ Efeito de horário de aplicação $(\mathrm{H})$} \\
\hline Diurna & $75,42 \mathrm{~b}^{\underline{1}}$ & $75,17 \mathrm{~b}$ & $76,33 \mathrm{~b}$ & $85,58 \mathrm{~b}$ & $87,58 \mathrm{~b}$ & $91,17 \mathrm{~b}$ & $87,50 \mathrm{~b}$ & $73,33 \mathrm{~b}$ & $64,33 \mathrm{~b}$ \\
\hline Noturna & $82,33 \mathrm{a}$ & $82,25 \mathrm{a}$ & $90,92 \mathrm{a}$ & $96,67 \mathrm{a}$ & $98,25 \mathrm{a}$ & $98,83 \mathrm{a}$ & $98,67 \mathrm{a}$ & $97,42 \mathrm{a}$ & $94,17 \mathrm{a}$ \\
\hline \multicolumn{10}{|c|}{ Efeito de doses (D) } \\
\hline $0,1 \mathrm{~kg} \mathrm{ha}^{-1}$ & $66,67 \mathrm{c}$ & $69,33 \mathrm{~b}$ & $68,17 \mathrm{~b}$ & $78,33 \mathrm{~b}$ & $83,50 \mathrm{~b}$ & $87,50 \mathrm{~b}$ & $80,83 \mathrm{~b}$ & $66,50 \mathrm{~b}$ & $53,67 \mathrm{c}$ \\
\hline $0,2 \mathrm{~kg} \mathrm{ha}^{-1}$ & $81,50 \mathrm{~b}$ & $79,17 \mathrm{ab}$ & $86,67 \mathrm{a}$ & $95,50 \mathrm{a}$ & $95,83 \mathrm{a}$ & $96,67 \mathrm{ab}$ & $94,83 \mathrm{a}$ & $90,17 \mathrm{a}$ & $77,67 \mathrm{~b}$ \\
\hline $0,3 \mathrm{~kg} \mathrm{ha}^{-1}$ & $73,50 \mathrm{bc}$ & $73,50 \mathrm{~b}$ & $83,17 \mathrm{ab}$ & $91,17 \mathrm{ab}$ & $92,83 \mathrm{ab}$ & $96,17 \mathrm{ab}$ & $97,33 \mathrm{a}$ & $93,83 \mathrm{a}$ & $88,50 \mathrm{ab}$ \\
\hline $0,4 \mathrm{~kg} \mathrm{ha}^{-1}$ & $93,83 \mathrm{a}$ & $92,83 \mathrm{a}$ & $96,50 \mathrm{a}$ & $99,50 \mathrm{a}$ & $99,50 \mathrm{a}$ & $99,67 \mathrm{a}$ & $99,33 \mathrm{a}$ & $99,00 \mathrm{a}$ & $97,17 \mathrm{a}$ \\
\hline
\end{tabular}

1/ Médias acompanhadas de mesma letra, nas colunas, não diferem estatisticamente entre si pelo teste de Tukey (5\%).

aos 47, 54 e 61 dias após a aplicação. A aplicação noturna apenas proporcionou melhor controle que a diurna nas doses de 0,1 e $0,2 \mathrm{~kg} \mathrm{ha}^{-1}$ nas três épocas de avaliação. Na dose de $0,3 \mathrm{~kg} \mathrm{ha}^{-1}$, o controle do aguapé foi menor na aplicação diurna apenas na avaliação realizada aos 61 dias após a aplicação.

Na Tabela 7, também é importante observar que, nas aplicações noturnas, a eficácia de controle foi maior que na diurna e houve similaridade estatística entre as quatro doses testadas. Na aplicação diurna, as menores doses proporcionaram menores valores de controle, devido à mais intensa rebrota do aguapé.

Os resultados observados corroboram os obtidos no Brasil por Martins et al. (2002) e Neves et al. (2002), segundo os quais o diquat é eficiente no controle do aguapé, e acrescentam a informação de que doses mais baixas e aplicações diurnas favorecem a rebrota das plantas, como também observaram Neves et al. (2002).

Segundo Rodrigues \& Almeida (2005), o diquat é um herbicida de contato, não seletivo, que interfere no processo de captação de energia solar pela fotossintese. Na presença de luz, forma-se o poder redutor no cloroplasto mediante captação do elétron proveniente da redução da ferredoxina, o qual reduz o íon bipiridílio a radical bipiridílio. Este não fica na forma reduzida (instável) e, na presença de oxigênio e água, oxida-se novamente, voltando à forma anterior, liberando elétrons que, em presença de água e oxigênio, formam uma molécula de água oxigenada. Esta, em altas 
Tabela 7 - Valores médios de controle de Eichhornia crassipes determinados no desdobramento dos graus de liberdade das interações entre as variáveis principais de horário de aplicação e dose de diquat - segundo experimento

\begin{tabular}{|l|c|c|c|c|}
\hline \multirow{2}{*}{ Horário } & \multicolumn{4}{|c|}{ Dose de diquat } \\
\cline { 2 - 5 } & $0,1 \mathrm{~kg} \mathrm{ha}^{-1}$ & $0,2 \mathrm{~kg} \mathrm{ha}^{-1}$ & $0,3 \mathrm{~kg} \mathrm{ha}^{-1}$ & $0,4 \mathrm{~kg} \mathrm{ha}^{-1}$ \\
\hline \multicolumn{5}{|c|}{47 dias após aplicação } \\
\hline Diurna & $65,67 \mathrm{Bb}$ & $90,33 \mathrm{Aa}$ & $95,33 \mathrm{Aa}$ & $98,67 \mathrm{Aa}$ \\
\hline Noturna & $96,00 \mathrm{Aa}$ & $99,33 \mathrm{Aa}$ & $99,33 \mathrm{Aa}$ & $100,00 \mathrm{Aa}$ \\
\hline \multicolumn{5}{|c|}{54 dias após aplicação } \\
\hline Diurna & $40,00 \mathrm{Bc}$ & $81,67 \mathrm{Bb}$ & $89,67 \mathrm{Aab}$ & $98,00 \mathrm{Aa}$ \\
\hline Noturna & $93,00 \mathrm{Aa}$ & $98,67 \mathrm{Aa}$ & $98,00 \mathrm{Aa}$ & $100,00 \mathrm{Aa}$ \\
\hline \multicolumn{5}{|c|}{61 dias após aplicação } \\
\hline Diurna & $21,00 \mathrm{Bc}$ & $59,33 \mathrm{Bb}$ & $82,00 \mathrm{Bab}$ & $95,00 \mathrm{Aa}$ \\
\hline Noturna & $86,33 \mathrm{Aa}$ & $96,00 \mathrm{Aa}$ & $95,00 \mathrm{Aa}$ & $99,33 \mathrm{Aa}$ \\
\hline
\end{tabular}

Letras maiúsculas comparam médias para horários de aplicação dentro de doses; letras minúsculas comparam médias para doses dentro de horários de aplicação. Médias acompanhadas de mesma letra não diferem estatisticamente entre si

concentrações, causa a morte da planta. Esse processo somente ocorre em presença de luz.

Nas plantas tratadas no fim da tarde, ou durante a noite, não há produção de água oxigenada e os tecidos responsáveis pela translocação não são afetados, permitindo que o produto seja translocado e atinja parte mais interna da planta, antes de receberem luz e iniciar o processo oxidativo intenso. Essa é provavelmente a razão de a eficácia do herbicida ser maior quando a aplicação é feita no período noturno. No entanto, quando se utilizam doses altas, esse efeito pode ser compensado pela alta toxicidade do produto. Esse efeito também foi relatado por Neves et al. (2002), provavelmente pelo fato de os autores terem usado doses na faixa de 0,5 a 3,0 L de diquat por hectare. Nessas dosagens, não houve diferenças significativas no controle do aguapé entre as aplicações diurnas e noturnas.

Neves et al. (2002) ainda ponderam que o diquat evidencia rapidamente seus sintomas, o que pode constituir uma vantagem para aplicações em dias subsequentes, pois haverá facilidade de identificação das plantas tratadas. Essa observação é interessante, pelo fato de que as plantas de aguapé se movimentam ao sabor dos ventos e das correntes d'água, mesclando plantas tratadas e não tratadas, de um dia para o outro. Por outro lado, a ação rápida pode produzir grande quantidade de material passivel de decomposição em curto espaço de tempo, podendo induzir demanda biológica de oxigênio na coluna d'água, o que é ambientalmente indesejado.

\section{LITERATURA CITADA}

CARDOSO, L. R.; MARTINS, D.; TERRA, M. A. Sensibilidade a herbicidas de acessos de aguapé coletados em reservatórios do Estado de São Paulo. Planta Daninha, v. 21, n. 1, p. $61-67,2003$

CARVALHO, F. T. et al. Plantas aquáticas e nível de infestação das espécies presentes no reservatório de Barra Bonita, no rio Tietê. Planta Daninha, v. 21, n. 1, p. 15-19, 2003.

CARVALHO, F. T.; VELINI, E. D.; MARTINS, D. Plantas aquáticas e nível de infestação das espécies presentes no reservatório de Bariri, no rio Tietê. Planta Daninha, v 23, n. 2, p. 371-374, 2005.

HENARES, M. N. P. et al. Toxicidade aguda e efeitos histopatológicos do herbicida diquat na brânquia e no fígado da tilápia nilótica (Oreochromis niloticus). Acta Sci. Biol. Sci., v. 30, n. 1, p. 77-82, 2008 a.

HENARES, M. N. P. et al. Toxicidade agúda e efeitos histopatológicos do diquat na brânquia e no fígado do piauçu (Leporinus macrocechalus). Pesticidas, v. 17, p. 107-116, 2007.

HENARES, M. N. P. et al. Toxicidade aguda e histopatologia do herbicida Reward na brânquia e no fígado do pacu (Piaractus mesopotamicus). J. Braz. Soc. Ecotoxicol., v. 3, n. 1, p. $41-45,2008$ b.

HOLM, L. G. et al. The world worst weeds: distribution and ecology. Malabar: Krieger Publishing, 1991. 607 p.

JANA, S.; CHOUDHURI, M. A. Aquatic weed problems in West Bengal, India. Weeds Today, v. 14, p. 9, 1983.

LANGELAND, K. A.; SMITH, B. E. Evaluation of triclopyr and diquat for managing mixed populations of water hyacinth (Eichhornia crassipes) and water lettuce (Pistia stratiotes). In SOUTHERN WEED SCIENCE SOCIETY ANNUAL MEETING, 46., 1993, Charlote. Proceedings... Charlote: 1993. p. $250-254$.

LAVORENTI, A. Comportamento dos herbicidas no meio ambiente. In: WORKSHOP SOBRE BIODEGRADAÇÃO, 1996, Campinas. Anais... Jaguariúna: Embrapa-CNPMA, 1996. p. $81-92$

MARTINS, A. T.; PITELLI, R. A. Efeitos do manejo de Eichhornia crassipes sobre a qualidade da água em condições de mesocosmos. Planta Daninha, v. 23, n. 2, p. 233-242, 2005 
MARTINS, A. T. Diquat no manejo de aguapé Eichhornia crassipes (Mart.) Solms e seu impacto sobre fatores limnológicos. 2009. 110 f. Tese (Doutorado em Fitotecnia) Universidade Estadual de São Paulo, Botucatu, 2009.

MARTINS, D. et al. Controle químico de Pistia stratiotes, Eichhornia crassipes e Salvinia molesta em caixas d'água. Planta Daninha, v. 20, p. 83-88, 2002. (Edição Especial)

MARTINS, D. et al. Levantamento da infestação de plantas aquáticas em Porto Primavera antes do enchimento final do reservatório. Planta Daninha, v. 27, p. 879-886, 2009.

MARTINS, D. et al. Caracterização da comunidade de plantas aquáticas de dezoito reservatórios pertencentes a cinco bacias hidrográficas do Estado de São Paulo. Planta Daninha, v. 26, n. 1, p. 17-32, 2008
NEVES, T.; FOLONI, L. L.; PITELLI, R. A. Controle químico do aguapé (Eichhornia crassipes). Planta Daninha, v. 20, p. 89-97, 2002. (Edição Especial)

PITELLI, R. L. C. M. et al. Utilização de análise multivariada e redes neurais artificiais na determinação do comportamento de colonização de populações de macrófitas aquáticas no reservatório de Santana. Planta Daninha, v. 27, n. 3, p. 429-439, 2009.

PITELLI, R. A.; MARTINS, D.; VELINI, E. D. Interferência e controle de macrófitas aquáticas. In: VARGAS, L; ROMAN, E. R. Manual de manejo de controle de plantas daninhas. Passo Fundo, Embrapa, 2008. p. 299-328.

RODRIGUES, B. N.; ALMEIDA, F. S. Guia de herbicidas. 5.ed. Londrina: Grafmarke, 2005. 591 p. 